\title{
An investigation of several finiteness criteria relative to Zermelo-Fraenkel set theory
}

\author{
John Llewellyn Hickman
}

This thesis investigates the logical strengths and interrelationships of several finiteness criteria against a strict background of ZermeloFraenkel set theory.

The fact that all criteria discussed in this thesis are logically equivalent under the influence of even a weak choice principle somewhat obscures the veriety of concepts that are being employed. These criteria are classified by means of their logical strengths, and the characteristics of each resulting class are examined.

It is also shown that a fairly plausible generalization of one of these criteria does not satisfy the intuitive concept of finiteness.

Throughout this thesis, the required models of set theory are constructed by the boolean-valued technique.

Part of the results have appeared in this BULLETIN; see [1], [2].

References

[1] J.L. Hickman, "Some definitions of finiteness", Buzl. Austral. Math. Soc. 5 (1971), 321-330.

[2] J.L. Hickman, "Some definitions of finiteness: Corrigenda", BulZ. Austral. Math. Soc. 6 (1972), 319.

Received 14 January 1972. Thesis submitted to the Flinders University of South Australia, October 1971. Degree approved, June 1972. Supervisor: Dr R.J. Baxter. 